\title{
The influence of acute pulmonary embolism on early and delayed prognosis for patients with chronic heart failure
}

\author{
Leszek Gromadziński ${ }^{1,2}$, Ryszard Targoński ${ }^{3}$ Beata Januszko-Giergielewicz ${ }^{1,2}$, \\ Michał Ciurzyński ${ }^{4}$, Piotr Pruszczyk ${ }^{4}$ \\ ${ }^{1}$ Department of Internal Medicine, Gastroenterology and Hepatology, University Hospital, \\ University of Varmia and Masuria, Olsztyn, Poland \\ ${ }^{2}$ Department of Internal Medicine, Cardiology and Nephrology, Hospital, Ostroda, Poland \\ ${ }^{3}$ Department of Internal Medicine and Cardiology, Municipal Hospital, Olsztyn, Poland \\ ${ }^{4}$ Department of Internal Medicine and Cardiology, Warsaw Medical University, Warsaw, Poland
}

\begin{abstract}
Background: Patients with acute pulmonary embolism (APE) with concomitant chronic heart failure (CHF) are characterized by higher mortality rates than APE patients without $C H F$. The aim of this study is to evaluate the potential impact of APE on early and long-term prognosis in patients with $\mathrm{CHF}$.

Methods: This study included 87 patients with CHF with suspected APE. Patients were divided into two groups according to spiral computed tomography results: one group with confirmed APE and one with excluded APE. Total and cardiovascular mortality in patients of both groups during a 6-and 36-month follow up period was assessed. Potential risk factors for mortality in patients with CHF in short and long-term observations were identified.
\end{abstract}

Results: APE was diagnosed in 35 patients, and excluded in the remaining 52 patients. Total and cardiovascular 6-month mortality was higher in APE patients than in patients without APE: $34.3 \%$ and $28.5 \%$ vs. $13.4 \%$ and $11.5 \%, p=0.02, p=0.02$, respectively. In 6-month follow-up the only independent risk factor for mortality was the presence of APE $(H R=2.7,95 \%$ CI 1.1-24.4, $p=0.04)$. However, in the 36-month follow-up APE had no effect on mortality.

Conclusions: Patients with CHF and acute episode of PE are characterized by a higher 6-month total and cardiovascular mortality rate following discharge from hospital compared to patients hospitalized due to acute CHF decompensation. Moreover, recent episode of PE in patients with CHF is an independent risk factor for early mortality in a 6-month follow-up. (Cardiol J 2012; 19, 6: 625-631)

Key words: heart failure, mortality, prognosis, pulmonary embolism

Address for correspondence: Leszek Gromadziński, MD, PhD, Department of Internal Medicine, Gastroenterology and Hepatology, University Hospital, University of Varmia and Masuria, ul. Warszawska 30, 10-082 Olsztyn, Poland, tel: +48 8952453 89, e-mail: lgol@op.pl

Received: 11.07.2012 Accepted: 06.09.2012 
Table 1. Etiology of chronic heart failure (CHF) in both studied groups.

\begin{tabular}{lcccc}
\hline Causes of CHF (\%) & All patients $(\mathbf{n}=\mathbf{8 7})$ & PE+ (n= 35) & PE- (n = 52) & P \\
\hline Coronary artery disease* $^{*}$ & $27(32.9)$ & $9(25.7)$ & $18(34.6)$ & 0.37 \\
Hypertension & $33(37.9)$ & $15(42.8)$ & $18(34.6)$ & 0.43 \\
Atrial fibrillation & $10(11.5)$ & $5(14.2)$ & $5(9.6)$ & 0.50 \\
Valvular heart disease & $7(8.0)$ & $4(11.4)$ & $3(5.7)$ & 0.34 \\
Dilated cardiomyopathy & $10(11.5)$ & $2(5.7)$ & $8(15.3)$ & 0.16 \\
HFPEF (LVEF > 45\%) & $49(56.3)$ & $22(62.8)$ & $27(52.0)$ & 0.31 \\
\hline
\end{tabular}

*History of myocardial infarction, percutaneous transluminal coronary angioplasty and coronary artery bypass grafting, PE — pulmonary embolism; HEPEF - heart failure with preserved ejection fraction, LVEF - left ventricular ejection fraction

\section{Introduction}

Chronic heart failure (CHF) is a known and significant risk factor for acute pulmonary embolism (APE). Moreover, patients with APE and coexisting $\mathrm{CHF}$ are characterized by a higher mortality rate than APE patients without CHF [1]. In large $\mathrm{PE}$ registries, such as ICOPER registry, CHF was observed in $10.5 \%$ of patients with APE and was found to be an independent mortality risk factor with a hazard ratio (HR) of 2.4 [2]. Similar symptomatology of both diseases still hinders diagnosis of APE and the implementation of an appropriate treatment. In patients with advanced $\mathrm{CHF}$, prevalence of APE increases and significantly influences the length of hospitalization, frequency of re-hospitalization or frequency of early death $[3,4]$. In the available literature, there are few reports evaluating the influence of APE on the survival of patients with $\mathrm{CHF}$.

The aim of this study was a diagnostic evaluation of the influence of past APE on the early and delayed survival of patients with CHF.

\section{Methods}

\section{Patient population}

A prospective study included 87 consecutive patients with $\mathrm{CHF}$ admitted to hospital due to sudden resting dyspnoea. The suspicion of APE was determined additionally based on the intermediate clinical probability according to the Wells scale, positive result of D-dimer $>0.5 \mu \mathrm{g} / \mathrm{mL}$ assay measured with immunoturbobidimetric method LIATEST, and a maximal velocity of tricuspid valve regurgitation (TR) $>2.8 \mathrm{~m} / \mathrm{s}$. Etiology of CHF in both studied groups is shown in Table 1.

This study was approved by the Regional Bioethics Committee, and all patients provided informed consent.
Patients were divided into two groups according to the results of spiral computed tomography ( $\mathrm{sCT}$ ) of the thorax and compression ultrasound (CUS) of lower extremities veins. Pulmonary embolism was diagnosed when at least segmental thromboemboli were visualized. Six- and 36-month total mortality and cardiovascular (CV) mortality were evaluated. Analyzis of CV mortality included: myocardial infarction, sudden cardiac death, APE, decompensated CHF and cerebral stroke; whereas the analysis of total mortality comprised the remaining causes including the neoplastic disease. Data on patients deaths were obtained from the regional Department of National Health Fund. Also, potential and independent risk factors for the death of patients with CHF under short- and long-term observations were determined.

\section{Definitions}

$\mathrm{CHF}$ was diagnozed based on a clinical findings according to Framingham criteria and earlier medical records. APE was recognized when at least one filling defect at $\mathrm{SCT}$ was visualized in at least segmental arteries. APE was excluded in the case of a negative $\mathrm{SCT}$ result and a negative CUS result. CUS was carried out in 4 points within femoral and popliteal veins. The total collapse of a vessel wall excluded the presence of thrombosis; whereas, a lack of total collapse of the vessel confirmed the presence of proximal thrombosis [5, 6]. Renal failure was diagnozed when serum creatinine level was found to be $\geq 1.3 \mathrm{mg} / \mathrm{dL}$ on admission. Hyponatremia was diagnozed when sodium $(\mathrm{Na})$ level in blood serum was $<136 \mathrm{mmol} / \mathrm{L}$ on admission.

Patients with acute coronary syndromes within the last 14 days and patients without diagnozed CHF were not included in this study.

\section{Echocardiographic examination}

For all patients, a standard echocardiographic examination was performed with a $2.5 \mathrm{MHz}$ trans- 
ducer (System 5, Vingmed, General Electric). In parasternal long-axis projection, left ventricular end-diastolic dimension (LVEDD) and right ventricular end-diastolic dimension (RVEDD) were measured. In apical 4-chamber projection, maximal tricuspid regurgitation pressure gradient (TRPG) and left ventricular ejection fraction (LVEF) employing the simplified Simpson method were evaluated $[7,8]$. With the use of one-dimensional M-mode examination, a tricuspid annular plane systolic excursion (TAPSE) was measured [9].

\section{Treatment}

Patients with $\mathrm{CHF}$ received standard therapy for heart failure: angiotensin converting enzyme (ACE)-inhibitor or AT1 receptor antagonist, beta-receptors antagonist, diuretics or spironolactone. In some patients with persistent atrial fibrillation digoxin was used. Patients with confirmed APE were treated with intravenous infusion of non-fractionated heparin in a therapeutic dose under the control of activated partial thromboplastin time $50-70 \mathrm{~s}$ for at least 5 days; and in all patients oral anticoagulant was included in their treatment under the control of normalized prothrombin time (INR) of 2.0-3.0 value. Non-fractionated heparin was discontinued after 2 days INR $>2$. All patients with confirmed APE were discharged from hospital on anticoagulant treatment recommended for at least 3 months.

\section{Statistical analysis}

For comparison, Student's t test or Mann-Whitney test were used depending on the distribution of variables. Data on normal distribution were presented as a mean with standard deviation (SD). Variables not meeting the requirements of a normal distribution were presented as a median with a range of values; the $\chi^{2}$ test was used for comparison of variables in multi-partition tables. To identify predictors of mortality, Cox proportional hazards regression analysis was performed. Continuous variables were analyzed in the analysis of dichotomous form according to significant cut-off points. For multivariate analysis, only those parameters were included which demonstrated statistical significance $(\mathrm{p}<0.05)$ in univariate analysis. Survival time of patients was determined by the Kaplan-Meier curve; the log-rank test was used for comparison. Statistical analyzis was carried out with the use of Statistica 6 software.

\section{Results}

The study comprized 87 consecutive patients with $\mathrm{CHF}$ admitted to hospital due to episode of sudden dyspnoea at rest, with clinically suspected APE of at least a moderate clinical probability according to the Wells' probability scale [10], positive result of D-dimer $>0.5 \mu \mathrm{g} / \mathrm{mL}$ and TR $>2.8 \mathrm{~m} / \mathrm{s}$. Eventually, APE was tomographically diagnosed in 35 patients, while in the remaining 52 subjects APE was excluded. Demographic and clinical characteristics of patients in both groups are presented in Table 2.

The mean age of the patients in both groups, with confirmed APE and without APE, was similar. Incidence of concomitant diseases, such as: arterial hypertension, history of myocardial infarction, atrial fibrillation, chronic obstructive pulmonary disease was also similar for both groups.

CHF of NYHA class IV, III and II ware present in a similar percentage in both studied groups. However, at echocardiographic examination, patients with confirmed APE were characterized by lower LVDD, higher RVDD, decreased RV contractility assessed with TAPSE than patients with excluded APE. However, patients in both groups did not differ in TRPG value and LVEF. Plasma creatinine level was significantly higher in the APE group, although renal failure was present with a similar frequency in both groups, $43.3 \%$ of APE patients and in $31.2 \%$ of patients without APE, respectively $(\mathrm{p}=0.27)$. Venous thromboembolism (VTE) risk factors, the presence of the neoplastic disease, a history of surgery within the past month and a positive history of APE or venous thrombosis in both examined groups were similar. Treatment of $\mathrm{CHF}$ in both groups did not differ significantly with regard to the frequency of beta-receptors antagonists use, ACE inhibitors, AT1 receptors antagonists, diuretics, spironolactone and cardiac glycosides and is shown in Table 3. Obviously patients with diagnozed APE were anticoagulated and at discharge $85 \%$ of them were treated with oral anticoagulants according to INR, and remaining $15 \%$ were treated with weight adjusted dose of low molecular weight heparin. Patients without APE received an oral anticoagulant at discharge in $53 \%$ of cases. The presence of atrial fibrillation was the main indication for long term anticoagulant prophylaxis in these patients.

During a 6-month observation period, total mortality was found to be higher in a group of patients with confirmed APE when compared to patients without APE and was $34.4 \%$ vs. $13.4 \%$, respectively $(\mathrm{p}=0.02)$. However, at 36 -month follow-up, total mortality did not differ statistically between both groups, but the tendency for a higher mortality rate was observed in patients with 
Table 2. Demographic and clinical characteristics of patients of both groups.

\begin{tabular}{|c|c|c|c|c|}
\hline Variables & All patients $(n=87)$ & $P E+(n=35)$ & PE- $(n=52)$ & $\mathbf{P}$ \\
\hline Age (years) (SD) & $74.3 \pm 11.3$ & $75.7 \pm 10.9$ & $73.4 \pm 11.5$ & 0.35 \\
\hline Female gender (\%) & 51.7 & 54.3 & 50.0 & 0.17 \\
\hline Hypertension (\%) & 63.2 & 62.8 & 63.4 & 0.43 \\
\hline Prior MI (\%) & 31 & 22.8 & 36.5 & 0.17 \\
\hline Diabetes mellitus (\%) & 25.3 & 31.4 & 19.2 & 0.19 \\
\hline Atrial fibrillation (\%) & 43.7 & 37.1 & 48.0 & 0.31 \\
\hline COPD (\%) & 11.5 & 6.9 & 16.6 & 0.21 \\
\hline NYHA class IV (\%) & 29.9 & 22.9 & 34.6 & 0.24 \\
\hline NYHA class III (\%) & 59.7 & 68.6 & 53.9 & 0.16 \\
\hline NYHA class II (\%) & 10.4 & 8.5 & 11.5 & 0.65 \\
\hline LVEDD [cm] & $5.35 \pm 10$ & $5.09 \pm 1.0$ & $5.51 \pm 0.9$ & 0.05 \\
\hline LVEDD > $5.6 \mathrm{~cm}(\%)$ & 39.0 & 31.4 & 44.2 & 0.23 \\
\hline RVEDD [cm] & $3.3 \pm 0.5$ & $3.4 \pm 0.4$ & $3.2 \pm 0.6$ & 0.04 \\
\hline RVEDD > $3.0 \mathrm{~cm}(\%)$ & 77.0 & 88.5 & 67.3 & 0.02 \\
\hline TRPG [mm Hg] & $47 \pm 14$ & $50 \pm 14$ & $45 \pm 13$ & 0.10 \\
\hline TRPG > $3.4 \mathrm{~m} / \mathrm{s}(\%)$ & 43.7 & 48.5 & 38.4 & 0.34 \\
\hline LVEF (\%) & $47.8 \pm 15$ & $50 \pm 13$ & $46 \pm 16$ & 0.13 \\
\hline LVEF $<40 \%$ & 34.5 & 25.7 & 40.3 & 0.15 \\
\hline TAPSE $[\mathrm{cm}]$ & $1.5 \pm 0.4$ & $1.4 \pm 0.3$ & $1.6 \pm 0.4$ & $<0.0001$ \\
\hline Serum sodium $[\mathrm{mmol} / \mathrm{L}]$ & $139.2 \pm 5.2$ & $138.7 \pm 0.6$ & $139.5 \pm 4.5$ & 0.54 \\
\hline Serum sodium < $136 \mathrm{mmol} / \mathrm{L}(\%)$ & 27.3 & 37.9 & 21.2 & 0.11 \\
\hline Serum creatinine $[\mathrm{mg} / \mathrm{dL}]$ & $1.1(0.6-3.4)$ & $1.2(0.6-3.4)$ & $1.0(0.6-2.2)$ & 0.03 \\
\hline Serum creatinine $\geq 1.3 \mathrm{mg} / \mathrm{dL}(\%)$ & 35.9 & 43.3 & 31.2 & 0.27 \\
\hline Cancer (\%) & 11.5 & 11.4 & 11.5 & 0.98 \\
\hline Surgery within 1-month (\%) & 2.3 & 5.7 & 0 & 0.08 \\
\hline Previous VTE (\%) & 11.5 & 11.4 & 11.5 & 0.98 \\
\hline
\end{tabular}

PE — pulmonary embolism; MI — myocardial infarction; COPD — obstructive pulmonary disease; NYHA — New York Heart Association; LVEDD — left ventricular end-diastolic diameter; RVEDD — right ventricular end-diastolic diameter; TRPG — tricuspid regurgitation pressure gradient; LVEF — left ventricular ejection fraction; TAPSE — tricuspid annular peak systolic excursion; VTE — venous thromboembolism

Table 3. Treatment of chronic heart failure in both groups.

\begin{tabular}{lccc}
\hline Variables & $\begin{array}{c}\text { PE+ } \\
\text { (n }=35)\end{array}$ & $\begin{array}{c}\text { PE- } \\
\text { (n }=\mathbf{5 2})\end{array}$ & P \\
\hline Beta-blockers & $94 \%$ & $93 \%$ & 0.83 \\
ACEl/AT1 receptors & $73 \%$ & $87 \%$ & 0.10 \\
antagonists & & & \\
Diuretics & $60 \%$ & $71 \%$ & 0.32 \\
Spironolactone & $60 \%$ & $65 \%$ & 0.67 \\
Cardiac glycosides & $7 \%$ & $14 \%$ & 0.34 \\
Oral anticoagulant/LMWH & $85 / 15 \%$ & $53 / 0 \%$ & 0.003 \\
\hline
\end{tabular}

$\mathrm{PE}$ - pulmonary embolism; ACEI — angiotensin converting enzyme inhibitors; LMWH - low molecular weight heparin

APE, which was $51.4 \%$ vs. $36.5 \%$, respectively $(\mathrm{p}=0.11)$ (Fig. 1). Interestingly, in a 6-month observation, CV mortality was higher in patients with APE than in the group without APE and was $28.5 \%$ vs. $11.5 \%$, respectively $(\mathrm{p}=0.02)$; whereas in

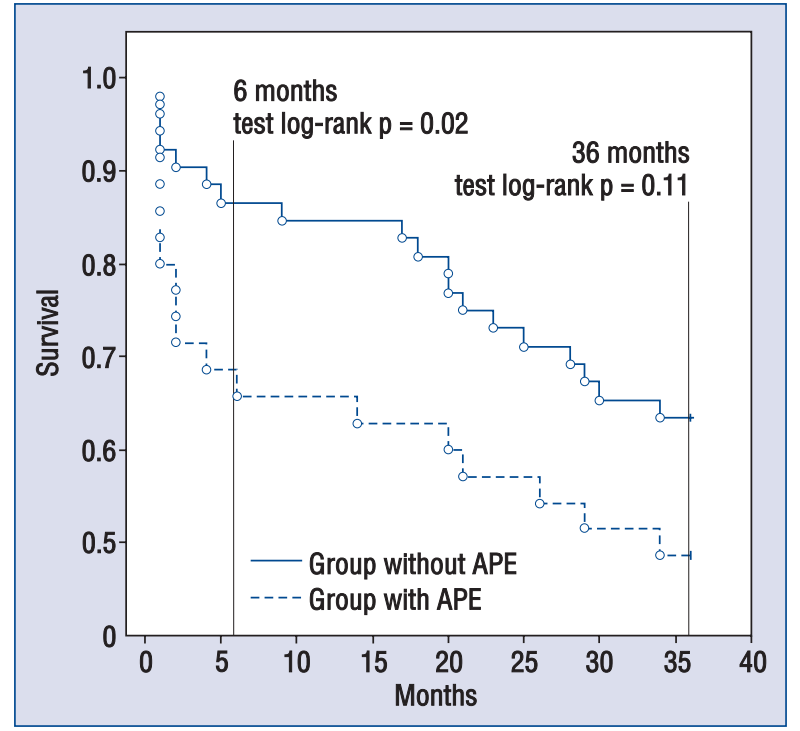

Figure 1. Survival estimated using Kaplan-Meier curves in patients with and without acute pulmonary embolism (APE) in a 36-month observation in analyzis of total mortality. 


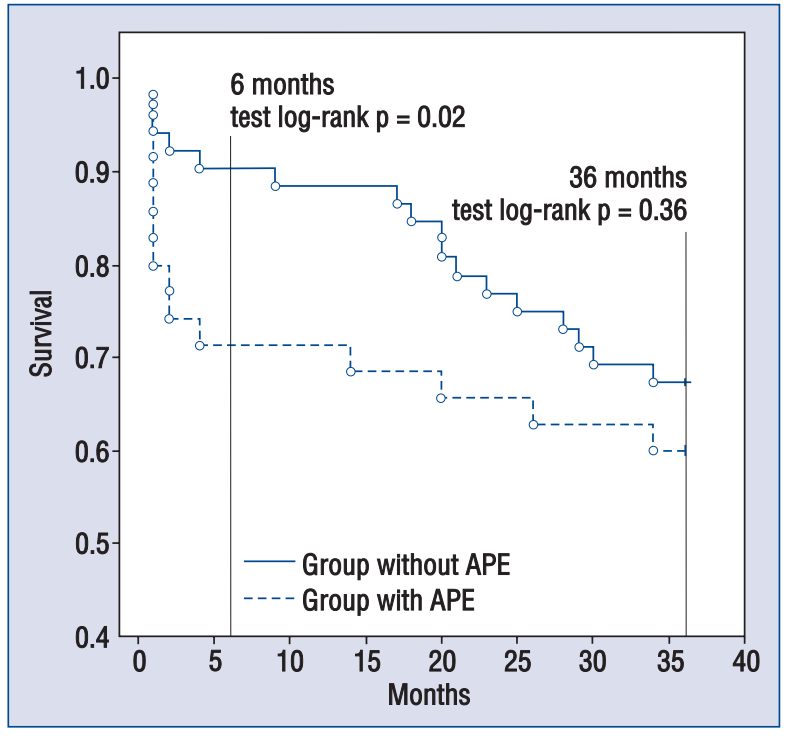

Figure 2. Survival estimated using Kaplan-Meier curves in patients with and without acute pulmonary embolism (APE) in a 36-month observation in analyzis of cardiovascular mortality.

a 36-month follow-up period, no such difference was observed (40.0\% vs. $34.6 \%, \mathrm{p}=0.36)$ (Fig. 2, Table 4).

In order to assess independent risk factors for the total mortality of patients with CHF, univariate and multivariate analyses of Cox proportional hazards regression analysis were performed. Potential significance of clinical, echocardiographic and laboratory parameters was analyzed. In a 6 -month observation period, mortality predictors of included: presence of APE: HR $=3.2(95 \%$ CI 1.3-12.2), $\mathrm{p}=0.02$, hyponatremia $(\mathrm{NaCl}<136 \mathrm{mmol} / \mathrm{L})$ : $\mathrm{HR}=2,8$ (95\% CI 1.3-18.5), $\mathrm{p}=0.03$ and renal failure (serum creatinine $\geq 1.3 \mathrm{mg} / \mathrm{dL}$ ): $\mathrm{HR}=2.6$ (95\% CI 1.2-17.4), $\mathrm{p}=0.04$ (Table 5).

However, in a multivariate analysis, the only independent risk factor for death in a 6-month observation period was the presence of APE with $\mathrm{HR}=2.7(95 \%$ CI 1.1-24.4), $\mathrm{p}=0.04$, while hyponatremia and renal failure were not independent
Table 5. Univariate predictors of death of patients with chronic heart failure at 6 months.

\begin{tabular}{lcc}
\hline Variables & HR $(95 \% \mathrm{CI})$ & $\mathbf{P}$ \\
\hline $\begin{array}{l}\text { Sodium serum level } \\
<136 \mathrm{mmol} / \mathrm{L}\end{array}$ & $2.8(1.3-18.5)$ & 0.03 \\
$\begin{array}{l}\text { Creatinine serum level } \\
\geq 1.3 \mathrm{mg} / \mathrm{dL}\end{array}$ & $2.6(1.2-17.4)$ & 0.04 \\
$\begin{array}{l}\text { Acute } \mathrm{pulmonary} \\
\text { embolism }\end{array}$ & $3.2(1.3-12.2)$ & 0.02 \\
\hline
\end{tabular}

$\mathrm{HR}$ - hazard ratio; $\mathrm{Cl}$ - confidence interval

Table 6. Multivariate predictors of death of patients with chronic heart failure at 6 months.

\begin{tabular}{lcc}
\hline Variables & HR $(95 \% \mathrm{CI})$ & $\mathbf{P}$ \\
\hline $\begin{array}{l}\text { Sodium serum level } \\
<136 \mathrm{mmol} / \mathrm{L}\end{array}$ & $2.2(0.9-15.9)$ & 0.10 \\
$\begin{array}{l}\text { Creatinine serum level } \\
\geq 1.3 \mathrm{mg} / \mathrm{dL}\end{array}$ & $2.2(0.8-15.7)$ & 0.11 \\
$\begin{array}{l}\text { Acute } \mathrm{pulmonary} \\
\text { embolism }\end{array}$ & $2.7(1.1-24.4)$ & 0.04 \\
\hline
\end{tabular}

$\mathrm{HR}$ - hazard ratio; $\mathrm{Cl}$ - confidence interval

mortality predictors (Table 6). In a long-term 36-month follow-up period, not found the potential risk factor for death of patients with $\mathrm{CHF}$.

\section{Discussion}

Decompensated chronic heart failure is a frequent cause of CV hospitalization [11-15].

Recent registries demonstrated significant inhospital mortality of CHF patients of $4-7 \%[16,17]$. It is generally accepted that patients with CHF are characterized by an increased risk of VTE. In various studies, the incidence of APE or deep vein thrombosis in the population of patients hospitalized due to CHF ranged from $4 \%$ to $14 \%$ [18-20]. In another study, the incidence of APE in a group of patients with $\mathrm{CHF}$ was $9.1 \%$ despite adequate use

Table 4. Total and cardiovascular mortality of patients of both groups.

\begin{tabular}{lcccc}
\hline Variables & All patients $(\mathbf{n}=\mathbf{8 7})$ & PE+ (n= 35) & PE- (n = 52) & P \\
\hline Total mortality 6-month (\%) & 21.9 & 34.3 & 13.4 & 0.02 \\
Total mortality 36-months (\%) & 42.5 & 51.4 & 36.5 & 0.11 \\
Cardiovascular mortality 6-months (\%) & 18.4 & 28.5 & 11.5 & 0.02 \\
Cardiovascular mortality 36-months (\%) & 36.8 & 40.0 & 34.6 & 0.46 \\
\hline
\end{tabular}

PE — pulmonary embolism 
of antithrombotic prophylaxis in $2 / 3$ of patients [3]. In the ICOPER registry with 2554 patients, APE accompanying CHF was diagnosed in $10.5 \%$ of patients [2]. The presence of CHF in patients with APE worsens their prognosis and is an independent risk factor for death [1,2]. Darze et al. [4] observed that APE worsens the prognosis or increases the frequency of hospitalization in a 3-month observation in patients with CHF. In our study, we compared patients with $\mathrm{CHF}$ and concomitant APE with patients with $\mathrm{CHF}$ and excluded APE in short- and long-term observations. In a 6 -month observation, the total and CV mortality of patients with APE was significantly higher compared to patients without APE: $34.4 \%$ vs. $13.4 \%(\mathrm{p}=0.02)$ and $28.5 \%$ vs. $11.5 \%(\mathrm{p}=0.02)$, respectively. However, the 36-month total and CV mortality for both studied groups was similar. It partly confirms the results of the Darze et al. study [4], which showed a trend towards a higher 3-month mortality rate for patients with $\mathrm{CHF}$ with confirmed APE compared to patients with CHF. In that study, a combination of 3-month mortality with 3-month re-hospitalization showed a significant predominance of adverse events in patients with APE by $72.2 \%$ vs. $43.5 \%$, respectively $(\mathrm{p}=0.02)$ [4]. In our study, total and CV mortality was evaluated and demonstrated a significant difference in frequency against patients with confirmed APE in a 6-month observation. To our knowledge, this is the first study which compares patients with CHF with confirmed APE during hospitalization with patients with $\mathrm{CHF}$ and excluded APE. In the Darze et al. study [4], a group of 18 patients with confirmed APE was compared with a group of 180 patients of whom only in 18 cases APE was excluded based on a negative result of $\mathrm{sCT}$ or pulmonary scyntygraphy. In that study, an independent factor for death or 3-month re-hospitalization was a history of APE (OR $=4.0,95 \%$ CI 1.1-15.1, $\mathrm{p}=0.038$ ). That study confirms our results that an independent risk factor for death of patients with CHF is a history of APE in a 6-month observation $(\mathrm{HR}=2.7,95 \%$ CI 1.1-24.4, $\mathrm{p}=0.04)$.

Persistent higher mortality of patients with $\mathrm{CHF}$ and APE during a 6-month period following discharge from hospital compared to patients without APE may be explained by its adverse influence on the clinical course of patients with CHF. A sudden increase of pulmonary pressure observed during APE results in pressure and volume overload of the RV. In previous studies, it was reported that prognosis for patients after APE depends mostly on RV efficiency and recurrences of APE. In our study, all patients with APE were at a low risk of early death and received prophylactic treatment with an oral anticoagulant in $85 \%$ of cases or low molecular weight heparin in $15 \%$ of cases. Despite an appropriate anticoagulant therapy, patients with a history of APE were characterized by a higher total and $\mathrm{CV}$ mortality rate than patients without APE in a 6-month observation period following discharge from hospital. Similar risk factors for VTE and acute coronary syndrome have been discussed recently. Can similar mechanisms of venous and arterial thrombosis cause an increase in the frequency of APE events and an increase in thromboembolic complications frequency after APE? Such a thesis is quite bold at present.

If the results of our study, indicating that APE is an independent factor for poor prognosis of patients with $\mathrm{CHF}$, are confirmed in further analyzes, we should consider a simple and efficient model of APE diagnosis in patients with $\mathrm{CHF}$ and a more efficient secondary anticoagulant prophylaxis following discharge from hospital.

\section{Limitations of the study}

The main limitation of our study is the fact that this one-center observation which was carried out with a relatively small group of patients.

\section{Conclusions}

Patients with CHF and acute episode of pulmonary embolism are characterized by a higher 6-month total and CV mortality rate following discharge from hospital compared to patients hospitalized due to acute CHF decompensation. Moreover, recent episode of $\mathrm{PE}$ in patients with $\mathrm{CHF}$ is an independent risk factor for early mortality in a 6 -month follow-up; whereas in a 36-month observation, past APE had no influence on mortality.

\section{Acknowledgement}

The authors of this manuscript have certified that they have complied with the Principles of Ethical Publishing present in the Declaration of Helsinki and that the study protocol was approved by a local ethics committee.

Conflict of interest: none declared

\section{References}

1. Monreal M, Munoz-Torrero JF, Naraine VS et al. RIETE Investigators. Pulmonary embolism In patients with chronic obstructive pulmonary disease or congestive heart failure. Am J Med, 2006; 119: 851-858. 
2. Goldhaber S, Visani L, de Rosa M. Acute pulmonary embolism: Clinical outcomes in the International Cooperative Pulmonary Embolism Registry (ICOPER). Lancet, 1999; 353: 1386-1389.

3. Darze ES, Latado AL, Guimaraes AG et al. Incidence and clinical predictors of pulmonary embolism in severe heart failure patients admitted to a coronary care unit. Chest, 2005; 128: 2576-2580 .

4. Darze ES, Latado AL, Guimaraes AG et al. Acute pulmonary embolism is an independent predictor of adverse events in severe decompensated heart failure patients. Chest, 2007; 131: 1838-1843.

5. Kearon C, Ginsberg JS, Hirsh J. The role of ultrasonography in the diagnosis of suspected deep venous thrombolism and pulmonary embolism. Ann Intern Med, 1998; 129: 1044-1049.

6. Perrier A, Bounameaux H. Ultrasonography of leg veins in patients suspected of having pulmonary embolism. Ann Intern Med, 1998; 128: 243-245.

7. Lang RM, Bierig M, Devereux RB et al. Recommendations of chamber quantification: A report from the American Society of Echocardiography's Guidelines and Standards Committee and the Chamber Quantification Writing Group, developed in conjunction with the European Association of Echocardiography, a branch of the European Society of cardiology. J Am Soc Echocardiogr, 2005; 18: 1440-1463.

8. Quinones MA, Otto CM, Stoddard M, Waggoner A, Zoghbi WA. Recommendations for quantification of Doppler echocardiography: A report from the Doppler Quantification Task Force of the Nomenclature and Standards Committee of the American Society of Echocardiography. J Am Soc Echocardiogr, 2002; 15: 167-184.

9. Kaul S, Tei C, Hopkins JM, Shah PM. Assessment of right ventricular function using two-dimensional echocardiography. Am Heart J, 1984; 107: 526-531.

10. Wells PS, Anderson DR, Rodger M et al. Derivation of a simple clinical model categorize patients probability of pulmonary embolism: Increasing the models utility with the SimpliRed D-dimer. Thromb Haemost, 2000; 83: 416-420.
11. Rosamond W, Flegal K, Friday G et al. Heart disease and stroke statistics-2007 update: A report from the American Heart Association Statistics Committee and Stroke Statistics Subcommittee. Circulation, 2007; 115: e67-e171.

12. Fang J, Mensah GA, Croft JB, Keenan NL. Heart failure-related hospitalization In the US,1979 to 2004. J Am Coll Cardiol, 2008; 52: $428-434$.

13. Jaarsma T, Haaijer-Ruskamp FM, Sturm H, Van Veldhuisen DJ. Management of heart failure in the Netherlands. Eur J Hear Fail, 2005; 7: 371-375.

14. Jessup M, Brozena S. Heart failure. N Engl J Med, 2003; 348: 2007-2018.

15. O'Connor CM, Stough WG, Gallup DS, Hasselblad V, Gheorghiade M. Demographics, clinical characteristics, and outcomes of patients hospitalized for decompensated heart failure: Observations from the IMPACT-HF registry. J Card Fail, 2005; 11: 200-205.

16. Baker DW, Einstadter D, Thomas C, Cebul RD. Mortality trends for 23,505 Medicare patients hospitalized with heart failure in Northeast Ohio, 1991to1997. Am Heart J, 2003; 146: 258-264.

17. Fonarow GC, Adams KF Jr, Abraham WT, Yancy CW, Boscardin WJ. Risk stratification for in-hospital mortality in acutely decompenased heart failure: classification and regression tree analysis. J Am Med Assoc, 2005; 293: 572-580.

18. Alikhan R, Cohen AT, Combe S et al. Prevention of venous thromboembolism in medical patients with enoxaparin: A subgroup analysis of the MEDENOX study. Blood Coagul Fibrynol, 2003; 14: 341-346.

19. Leizorovicz A, Cohen AT, Turpie AG, Olsson CG, Vaitkus PT, Goldhaber SZ. Randomized, placebo-controlled trial of dalteparin for the prevention of venous thromboembolism in acutely ill medical patients. Circulation, 2004; 110: 874-879.

20. Cohen AT, Davidson BL, Gallus AS et al. Efficacy and safety of fondaparinux for the prevention of venous thromboembolism in older acute medical patients: Randomised placebo controlled trial. BMJ, 2006; 332: 325-329. 